\title{
Notizen zu einer Skizze einer peirceanischen Theorie des Unbewussten ${ }^{1,2}$
}

\author{
Vincent Colapietro (State College, PA)
}

Zusammenfassung: Einer der ersten Autoren, der die Peirce'schen Gedanken für die Psychoanalyse fruchtbar zu machen versuchte, ist der amerikanische Philosoph Vincent Colapietro. Wir stellen unseren Lesern die fast schon klassisch zu nennende Arbeit von 1995 vor. Die Übersetzung stammt von Vera Saller. Colapietro zeigt auf, wie eine Semiotik des Unbewussten nach Peirce aussehen könnte. Peirce und Freud stimmen darin überein, dass weite Teile des menschlichen Denkens unbewusst ablaufen. Der Autor weist nach, dass Peirce nicht nur das, was bei Freud als Vorbewusstes bezeichnet wird, anerkennt, sondern auch ein dynamisches Unbewusstes mit den Mechanismen der Verdrängung und der Zensur vorgesehen hat. Er erläutert das Feld von Gewohnheitsbildung und Bewusstsein, um dann schliesslich das Freud'sche Unbewusste als Phänomen im Peirce'schen Sinn gemäss den drei Kategorien zu analysieren.

Schlüsselwörter: Semiotik des Unbewussten, Vorbewusstes, Unbewusstes und Gewohnheit, Firstness der Thirdness, kompetente Akteure, Unbewusstes als Phänomen.

Obwohl Peirce offensichtlich unbewusste seelische Vorgänge anerkannte, hat er diese Dimension nicht systematisch oder tiefergehend untersucht. Ebenso wenig hat er (soweit ich in der Lage war, festzustellen) in irgendeiner Weise, und sei es nur in Form einer flüchtigen Skizze, erhellt, was für eine Bedeutung jenen Phänomenen, die gewöhnlich unter dem Begriff des Unbewussten gefasst werden, im Rahmen seiner allgemeinen Theorie der Zeichen zukommen würde. Dem Forscher, der versucht zu ergründen, wie Peirce sich dem Unbewussten angenähert oder das Bewusstsein ergründet hat, stellt sich deshalb eine vierfache Aufgabe. Dieser Forscher müsste 1. Peirce' eigene verstreute Bemerkungen über das Unbewusste und verwandte Themen wie Rationalisierung, Selbst-Betrug und Hypnose zusammenstellen. 2. Fruchtbare Wege vorschlagen, wie die wichtigsten Peirce'schen Thesen (vor allem seine Kategorien und seine Theorie der Zeichen) mit dem Unbewussten zu verbinden seien. 3. Eine explizit semiotische Interpretation des Unbewussten formulieren. 4. Dieses Verständnis des Unbewussten sollte mit 
jenem Sigmund Freuds, Carl Gustav Jungs, Jacques Lacans und/oder Julia Kristevas verglichen werden. Ich bin weit davon entfernt, diese Aufgabe bewältigt zu haben, aber ich bin in meinen Recherchen genügend weit vorgedrungen, um meine Notizen dazu, wie ich das Peirce'sche Unbewusste skizzieren würde, zu präsentieren. In dieser Arbeit werde ich mich auf Teile der ersten beiden Aufgaben beschränken. Ganz im Geiste von Peirce ist die Rechtfertigung, diese Notizen zu publizieren, heuristisch:Sie sollen andere Forscher anleiten und anspornen, Untersuchungen in einem bis dahin vernachlässigten, aber wichtigen Feld zu unternehmen. Ich erhoffe mir auch Reaktionen auf meinen Aufsatz, die meine eigene Arbeit vorantreiben.

Dass ich eben zuvor von Lacan sprach, erinnert mich zu erwähnen, dass es in der Tradition der Saussure'schen Semiologie bereits eine ausgearbeitete Theorie des Unbewussten gibt, während wir in der Peirce'schen Tradition nichts Vergleichbares finden. Es mag sein, dass das Unbewusste wie eine Sprache strukturiert ist, wie Lacan (1977) behauptet; aber die Bedeutung dieser Behauptung hängt vollständig davon ab, was man unter der Struktur einer Sprache versteht (vgl. Shapiro, 1983, auch Pharies, 1985). Oder spezifischer: Sprache als ein System rein formaler Unterschiede $\mathrm{zu}$ fassen ist etwas ganz anderes, als sie als ein Netz konkreter, verkörperter Gewohnheiten ${ }^{3}$ zu verstehen (Colapietro, 1989; 1991, de Lauretis, 1984; 1994). Dieser und weitere Unterschiede verweisen direkt darauf, dass Peirce die Behauptung, das Unbewusste sei wie eine Sprache strukturiert, sehr anders auslegen würde als Lacan. Aber bevor aufschlussreiche Vergleiche gezogen oder konstruktive Differenzen ausgemacht werden, soll die Peirce'sche Perspektive für sich genommen erläutert werden.

Bevor ich beginne, sind indessen zwei weitere, letzte Vorbemerkungen angebracht. Da sind zum einen die Ausführungen von Lucia Santaella Braga (1993), die kürzlich eine Liste der ernsthaften Schwierigkeiten aufstellte, die sich bei der Anwendung von Peirce' Semiotik ergeben, und die fruchtbarsten Strategien im Umgang mit diesen aufzeigte. Ich habe mich bemüht, ihren hermeneutischen und heuristischen Kommentaren zu folgen, im Besonderen ihrem Rat «die intellektuelle Schwäche zu vermeiden, die allgemein auftritt, wenn die Peirce'schen Definitionen und Klassifikationen aus ihrer gegenseitigen Verbundenheit getrennt werden, die ihnen ihre Tragweite geben» (S. 405; vgl. auch Freuds Aussagen von 1930/1982d, S. 269). Wir haben das Auftreten dieser intellektuellen Schwäche zu vermindern versucht, indem wir uns für die formalen und dynamischen Geschehnisse bewusst auf das kategoriale Schema ${ }^{4}$ von Peirce verlassen haben (S. 407-408; vgl. Savan, 1987/88, sowie Shapiro, 1983). 
Zweitens scheint es angebracht, hier auf eine bedeutende Ähnlichkeit von Freud und Peirce (vielleicht nur eine unter vielen, indes eine der wichtigen) zu verweisen, wenn auch nur en passant. Wie Wollheim sagt, «stützte sich Freud [...] auf zwei heuristische Prinzipien. Da war einmal die Überzeugung vom sinnvollen Charakter menschlichen Handelns. «Gewinn an Sinn und Zusammenhang ist aber ein vollberechtigtes Motiv, das uns über die unmittelbare Erfahrung hinaus führen darf [1982a, S. 126]. Zweitens schätzte Freud alle Postulate umso mehr, je mehr sie sich verallgemeinern liessen; und er war misstrauisch gegenüber jeglichen Annahmen, im Mass wie sie ad hoc schienen und wie sie die Allgemeingültigkeit der Aussagen einschränkten $»^{5}$ (Wollheim, 1971, S. 195-196; 1972, S. 152). Natürlich weisen auch Peirce' Gedanken in die Richtung von beidem: Bedeutungsdichte und Allgemeinheit; grosszügige Konstruktion des Bereiches der bedeutenden ${ }^{6}$ Phänomene und das Bemühen, den Implikationen seiner eigenen Kategorien streng nachzukommen. Aber wie für Freud sollte auch für Peirce die Allgemeinheit der Aussagen nicht um den Preis der Spezifität gewonnen werden. Er insistiert: «Gedanken müssen leben und unendlich in neue und höhere Übersetzungen wachsen. Oder es zeigt sich, dass es keine genuine Gedanken sind» (CP 5.594) ${ }^{7}$. Aber unmittelbar danach stellt er fest: «Der Geist verliert sich in solch allgemeinen Fragen und er scheint in eine grenzenlose Leere zu gleiten» (ebd.). Um dies abzuwenden und um gleichzeitig den Gang der Forschung nicht zu behindern (indem man Wälle zwischen den Forschungsfeldern aufbaut, was hiesse, die Allgemeinheit beeinträchtigen), ist es entscheidend zu erkennen, dass das eigentliche Wesen von Denken und Telos ${ }^{8}$ in Richtung Spezifität und Konkretheit zielt, «genauso wie es auch wahr ist, dass es das Wesen der beiden ist, allgemein zu sein» und deshalb vermutlich auch vage (CP 5.594, für die Unterscheidung zwischen Allgemeinheit und Vagheit vgl. CP 5.450; 1970, S. 427, CP 5.505 ff.). Das Feld der Bedeutung ${ }^{9}$ grosszügig zu konstruieren bedeutet nicht, unverantwortlich jegliches Kriterium zu verwerfen, das bei konkurrierenden Deutungen den Wert der jeweiligen Deutung bemessen könnte; auch wenn potentiell alles bedeutsam sein kann, heisst das nicht, dass jede Deutung gleichermassen gültig sei (Eco, 1992, S. 144). Fantastische Überinterpretation ist nicht wünschenswerter als grenzenlose Leere (Eco, 1992). Eine Erläuterung dieser beiden heuristischen Prinzipien sehen wir in Peirce' expliziter Behandlung des Bewusstseins und - so möchte ich behaupten - in seinen grösstenteils impliziten Ausführungen zum Unbewussten. 


\section{Peirce über Geist und Bewusstsein}

«Die meisten von uns haben die Gewohnheit zu denken, Bewusstsein und psychisches Leben seien ein und dasselbe und überschätzen die Funktionen des Bewusstseins stark» (CP 6.489). Im Gegensatz zu diesen beiden Tendenzen unterscheidet Peirce das Bewusstsein sorgfältig vom Geist, überdies misst er dem bewussten Selbst durchgehend eine eher bescheidene Rolle zu. Lassen Sie uns deshalb die beiden Punkte etwas genauer betrachten.

1902 machte Peirce geltend, dass «[Eduard] Von Hartmann, bereits 1869 [1968? Anm. V.C.] überzeugend bewiesen habe, dass ein unbewusster Geist existiert» (CP 7.366). Peirce betonte, dass es ein Fehler sei, die Psychologie als das Studium des Bewusstseins zu definieren: «Denn wenn Psychologie auf die Phänomene des Bewusstseins beschränkt sein sollte, bliebe die Schaffung von mentalen Verbindungen, das Annehmen von Gewohnheiten, welches den eigentlichen Marktplatz der Psychologie darstellt, ausserhalb ihrer Boulevards. Wenn von der Fachrichtung der Psychologie gesagt wird, dass der Grossteil ihrer Studien die Phänomene des Bewusstseins betrifft, ist das, wie wenn man das Wasser als das Studienobjekt des Ichthyologen definieren wollte» (CP 7.367). Eine mögliche Interpretation dieser Metapher ist, dass das Bewusstsein das Medium sei, in dem sich die Gewohnheiten formen. Aber wie auch immer diese Trope gedeutet wird, die Stossrichtung des Textes von Peirce ist klar: Das Hauptgeschäft der experimentellen Psychologie sollen nicht Bewusstseinsprozesse und -stadien sein, sondern die Bildung und das Funktionieren von Gewohnheiten.

In diesem Kontext umfassen die Gewohnheiten auch die Instinkte (vgl. CP 5.504), denn die Letzteren sind, wie Peirce selber anregt, entweder angeborene Dispositionen oder angeborene Tendenzen für die Annahme spezifischer oder unspezifischer Dispositionen: «Tierische Instinkte sind natürliche Dispositionen oder angeborene Bestimmungen der Natur des Individuums (wenn wir als seine «Natur` das bestimmen, was bewirkt, dass sein Verhalten so ist, wie es ist), die sich durch einen gewissen vereinheitlichten Quasi-Zweck in seinem Verhalten äussern» (CP 7.382, Anm. 19). (Für eine genauer ausgearbeitete Klassifikation der Instinkte durch Peirce siehe MS 1343.) Peirce fährt fort mit der Behauptung, dass das triebhaft bestimmte Verhalten bei Menschen kaum einmal sprunghaft sei und es immer möglich sei, sich dessen bewusst zu werden. Mehr noch, solange der menschliche Organismus nicht unter einem ausserordentlichen Stress steht, ist «das Verhalten immer teilweise kontrolliert durch die willentlich ausgeführte Imagination und Reflexion», und zwar so weit, dass die Handlung dem Organismus selber «vollkommen rational» (CP 7.381, Anm. 19) vorkommt. Obwohl die Annahme, dass die 
spontanen Impulse im Selbstbild des Organismus einer rationalen Kontrolle unterliegen, stark übertrieben ist, hat sie doch einen realen Kern: Rationale Kontrolle ist keine vollständige Täuschung.

Die höchste Errungenschaft des Geistes bringt die Bereitschaft mit sich, Gewohnheiten anzunehmen und wieder abzulegen (CP 6.614); der Geist selber ist ein vielschichtiges System komplex verbundener Gewohnheiten. Noch genauer: Der vernünftige Geist ist, "gerade wegen seines kindlichen Charakters ein unreifer, instinktiver Geist», der sich in Richtung grösseren Selbstbewusstseins und Selbstkritik entwickeln kann und der durch die reflexiven Tendenzen auch grössere Selbstkontrolle entwickelt (CP 7.381). Kurz, Unreife ist eine notwendige Bedingung für Rationalität in Peirce' Sinn (vgl. z. B. CP 5.440; 1970, S. 416; 5.419; 1970, S. 399). Gerade dass die menschlichen Instinkte vollständig oder zumindest zum überwältigenden Teil schwach sind und nur unsicher eine Richtung anzeigen, macht sie «unendlich plastisch»; diese Plastizität schliesst aus, dass sie je einen endgültigen Status erreichen, der eine weitere Möglichkeit für Verfeinerung ausschlösse (CP 7.381). Und um das Paradoxe an der Sache noch klarer herauszustellen: Vollständige Reife würde nichts weniger bedeuten als gestoppte Entwicklung. Und um die Sache noch weiter zu treiben, weiter als Peirce selber es scheinbar intendierte, erlauben Sie mir anzunehmen, dass der instinktive Geist gleichzeitig eine Menge vorzeitig gereifter sowie unüberwindlich unreifer komplexer Tendenzen ist, die sich in mancher Hinsicht gegenseitig unterstützen, sich aber auch ihrer Natur nach widersprechen. So besteht das kulturelle Kodieren unserer instinktiven Tendenzen gleichzeitig in einem Brüchigwerden vorzeitig festgelegter Tendenzen und in einer Regelung wilder, unreifer Impulse. Menschliche Instinkte zeigen weder starre Festigkeit noch vollständige Plastizität: Ihr Charakter und speziell ihre Stärke wurzeln in einer langen biologischen Geschichte, während ihre Entwicklung und gegenseitige Integration zeigt, dass sie vitale Kräfte einer relativ kurzen, aber tief umgestaltenden kulturellen Geschichte sind.

Die Instinkte - die Gewohnheiten, mit denen wir geboren werden erleichtern das Annehmen von Gewohnheiten in erheblichem Masse. Wenn meine Hypothese stimmt, dann bedeutet die Annahme von diesen oder jenen Gewohnheiten den Bruch etablierter Gewohnheiten und das Zähmen chaotischer Tendenzen. Aber auch abgesehen von instinkthaften Verhaltensdispositionen kann folgendes mit Sicherheit gesagt werden: Immer geht der Bruch mit einer Gewohnheit mit erhöhtem Bewusstsein einher, während die Aufnahme von neuen Gewohnheiten das Bewusstsein zum Schwinden bringt. In Peirce' eigenen Worten «geht das Bewusstsein zurück, sobald sich eine Verhaltensgewohnheit 
bildet und es erwacht erneut, wenn die Verhaltensgewohnheit wieder zerbricht» (1995, S. 233, CP 6.613). Und doch ist es in einigen Kontexten so, dass die Annahme einer Gewohnheit selber davon abhängt, dass wir uns uns selbst bewusst werden. Es ist eine der Haupteigenschaften des Bewusstseins, dass es im etymologischen Sinne ein Unwohlsein ${ }^{10}$ ist - ein Unwohlsein, das durch eine Dysfunktion verursacht wird. Es ist in der Tat genau diese Äusserungsform des Bewusstseins, auf die Peirce in seinen frühen pragmatischen Essays (am offensichtlichsten in «Die Festigung der Überzeugung» und «Wie unsere Ideen zu klären sind»), aber auch in seinen späteren pragmatistischen Texten, den Akzent setzte. Das Unwohlsein, das bei den Akteuren als Zweifel empfunden wird, wird als Zeichen verstanden, dass etwas fehlt. Der menschliche Organismus ist gefordert, solche und ungezählte andere Zeichen somatischer Art wie auch solche aus der Umwelt, zu deuten, um Halt zu finden in einer unvermeidlich gefahrvollen Welt. Das heisst also, dass das menschlicheWohlergehen und der Gebrauch der Zeichen untrennbar miteinander verbunden sind, denn menschliche Existenz und menschliche Semiose sind aus demselben Stoff.

Die Behauptung, dass die Annahme neuer Gewohnheiten den Grad des Bewusstseins heruntersetze, muss modifiziert werden. ${ }^{11}$ Diese Einschränkung ist wichtig, denn sie zeugt von einem fein abgestimmten Verständnis der Beziehung zwischen Gewöhnung und Aufmerksamkeit; mehr noch, sie lässt eine genauere Beschreibung von Peirce' wirklichen Einsichten zu. Es ist nicht nur so, dass das Aufnehmen von Gewohnheiten in einigen Fällen grosse Aufmerksamkeit (Bewusstheit) erfordert, sondern die Wirkung einiger Gewohnheiten kann in gewisser Hinsicht in nichts weniger bestehen, als der Wahrscheinlichkeit einer besonderen Aufmerksamkeit. So ist zum Beispiel jemand, der gewohnheitsmässig Anteil nimmt, dank seiner Gewohnheit, gegenüber Gefühlen, Gedanken und Angelegenheiten von andern aufmerksam. In diesem Fall würde also die Gewohnheit die Aufmerksamkeit nicht verschwinden lassen, sondern sie eher beleben. Aber sogar hier könnte man einwerfen, dass die zunehmende Gewöhnung der Aufmerksamkeit dieselbe verflacht, und dies arbeitet wieder gegen eine volle und gebührende Aufmerksamkeit, die jede einzelne Situation tatsächlich verlangt. Das heisst, sogar jene Gewohnheiten, die die Aufmerksamkeit hervorbringen und erleichtern, lassen in gewissem Sinne das Bewusstsein verschwinden.

DerVersuch, menschliches Verhalten als Zusammenspiel von Bewusstsein und Gewöhnung zu beschreiben, ist Teil der Psychologie, so jedenfalls hat Peirce diese Wissenschaft verstanden. Hingegen spricht derVersuch, Bewusstsein und das Erreichen von Gewöhnung in semiotischen Begriffen zu beschreiben (z. B. unser all- 
tagspraktisches Bewusstsein als komplex verknüpfte Folge indexikalischer Zeichen, vgl. z. B. CP 5.493; 1970, S. 490) oder unsere entwickelten und sich entwickelnden Gewohnheiten als affektive, energetische Interpretanten und kognitive Prozesse (vgl. z. B. 5.487 ff.; 1970, S. 484 ff.) zu deuten natürlich für Peirce' Unterfangen, alle und jegliche Erfahrung sub specie semiotica zu verstehen (Hardwick, 1977 [Hrsg.], S. 85-86; vgl. Eco, 1976, S. 27).

Wie zu erwarten sind also Psychologie und Semiotik eng verbunden. Die Einzelstudie $^{12}$ der Gewohnheitsbildung durch empfindungsfähige Organismen und das verallgemeinernde Studium des Funktionierens von Zeichen auf nahezu allen Ebenen (von der geologischen Formation bis zu theologischen Mythen, von Fossilien und physikalischen Verschiebungen bis hin zu Metaphern und literarischen Anspielungen) hängen voneinander ab, wenn auch nicht symmetrisch. «Natürlich sollen Psychologen ihre eigenen, unschätzbar wertvollen Studien der Funktionen der Zeichenentstehung und des Zeichengebrauchs vornehmen, und sie tun es auch! Ich halte diese Studien für unschätzbar wertvoll, obwohl sie kaum zu endgültigen Schlussfolgerungen kommen werden, solange nicht grundsätzlichere [Zeichen-]Studien ihre erste Ernte eingebracht haben» (MS 675, zitiert nach Fisch, 1986, S. 340). Um sich ihren Zielen wirkungsvoll zu nähern, muss die experimentelle Wissenschaft des Geistes (d.h. der Gewohnheits-Systeme) die Ergebnisse des allgemeinen Studiums der Semiose oder der Zeichenprozesse abwarten, obwohl die experimentellen Studien in der Zwischenzeit eigene, unverwechselbare Beiträge zu unserem Verständnis einer spezifischen Art der Semiose beisteuern.

Schon Jahrhunderte bevor der Mensch begann, den Geist experimentell zu untersuchen, haben sich Menschen auf das verlassen, was Peirce nicht zögert als instinktive Psychologie zu bezeichnen. Und diese ist nicht einfach eine nutzlose Spur einer primitiven Vergangenheit; auch wir stützen uns unausweichlich auf eine solche Psychologie. Mehr noch, es ist für uns sinnvoll, uns so zu verhalten: «Wir sollten diese natürlichen, instinktiven Vorstellungen des Geistes hoch schätzen, denn sie sind für uns in der Praxis sehr nützlich» (CP 7.409; vgl. CP 5.511). Diese «natürliche Psychologie wächst fast identisch in allen Menschen als natürliche Folge ähnlicher Erfahrungen die auf ähnliche Eigenschaften des Verstandes wirken» (CP 7.421). Peirce gesteht explizit zu, dass «es [...] nicht den geringsten Grund für die Annahme [gibt], dass diese natürliche Psychologie vollständig wahr sei; im Gegenteil, sie umfasst wahrscheinlich gewichtige Fehlschlüsse». Trotzdem soll «ihre wirklich weitreichende Kompetenz [...] anerkannt werden angesichts ihrer Bedeutung in der Lebensführung; denn, aufs Ganze gesehen ist der Mensch [d.h., Homo Sapiens, V.C.] unter solchen Glaubenssätzen gediehen» (ebd.). 
Peirce war auch der Meinung, dass: «[...] [wir] [...] die naive Vorstellung [haben], dass unsere Glaubenssätze hauptsächlich durch unseren bewussten Intellekt geprägt seien, aber es ist nicht so» (CP 7.456; Hervorhebung V. C.). «Unser tägliches Leben ist», gemäss Peirce, «voller Glaubenssätze, die unwillentlich geprägt worden sind. Es ist die Ichbezogenheit unseres Egos, oder [das bewusste] Feld [unserer willentlichen] Aufmerksamkeit, das sich uns aufdrängt, mit seiner hochdeutschen [High German], bescheidenen Überzeugung, dass wir, was auch immer wir wissen, ihr verdanken. Es ist [aber einfach] nicht so» (CP 7.447). Die Vermutung, dass dieser naive Glaube bis zu einem gewissen Grad instinktiv sei, leuchtet ein, denn die Annahme der Kontrolle unserer Handlungen und unseres Bewusstseins ist wohl sowohl ein biologischer Imperativ als auch ein kulturelles Ideal. Es ist zudem aufschlussreich, diesen Text neben eine berühmte Passage aus Freuds Vorlesungen zur Einführungen in die Psychoanalyse zu stellen:

[...] die heutige psychologische Forschung [...], welche dem Ich nachweisen will, dass es nicht einmal Herr ist im eigenen Hause, sondern aufkärgliche Nachrichten angewiesen bleibt von dem, was unbewusst in seinem Seelenleben vorgeht. Auch diese Mahnung zur Einkehr haben wir Psychoanalytiker nicht zuerst und nicht als die einzigen vorgetragen, aber es scheint uns beschieden, sie am eindringlichsten zu vertreten und durch Erfahrungsmaterial, das jedem einzelnen nahegeht, zu erhärten. Daher die allgemeine Auflehnung gegen unsere Wissenschaft [...] und die Entfesselung der Opposition von allen Zügeln unparteiischer Logik (Freud, 1982b, S. 284).

Nur, für Peirce ist das Mass, bis zu dem das Ich nicht einmal Herr im eigenen Haus ist, nicht so hoch, dass er jegliche Möglichkeit zur Selbst-Kontrolle ausschliessen würde (Colapietro, 1989). Damit ist auch der Grad, bis zu dem der unbewusste Geist sich vom Bewusstsein unterscheidet nicht so hoch, dass die beiden Teile hermetisch voneinander abgeriegelt wären. Angesichts Peirce' Engagement für den Synechismus (d.h. dem Prinzip der Kontinuität) weigerte er sich, eine vollständige, scharfe Trennungslinie zwischen bewussten und unbewussten Dimensionen der menschlichen Psyche zu ziehen. Das eine wirft seinen Schatten auf das andere. «Wir machen im Normalfall alle Unterscheidungen zu absolut» (CP 7.438), eingeschlossen jene zwischen Bewusstsein und Unbewusstem (vgl. Wollheim, 1971, S. 180; 1972, S. 142). 


\section{Das Unbewusste in seiner Unterscheidung vom Vorbewussten}

Hier ist es angebracht daran zu erinnern, dass Peirce geneigt war, das menschliche Bewusstsein als «einen unergründlichen See zu betrachten, dessen Wasser zwar durchsichtig erscheinen, der uns indessen nur an der Oberfläche auf kurze Distanz klar sehen lässt» (CP 7.547; vgl. auch CP 7.553; CP 7.554). Diese Sichtweise stellt die Vergleichbarkeit der beiden Konzepte in Frage, denn (in Freuds Begriffen) müssen wir nicht nur zwischen Bewusstem und Unbewusstem unterscheiden, sondern auch Unbewusstes von Vorbewusstem trennen. Erkennt Peirce ein Unbewusstes im strengen, freudianischen Sinn an, oder beziehen sich seine Äusserungen nur auf das Vorbewusste? Obwohl das eine schwierige terminologische Frage ist (vor allem auch, weil Freuds eigener Gebrauch der Termini sich verändert), gibt es doch Texte, in denen Peirce das Unbewusste klar als Gegensatz zum Vorbewussten beschreibt. So versichert er z. B.: «Vernunft ist ihrer Natur nach sehr egoistisch (...) Die Menschen stellen sich oft vor, dass sie der Vernunft folgen, während in Wirklichkeit die Gründe, die sie sich selbst zuschreiben, nichts als Entschuldigungen sind, welche der unbewusste Instinkt erfindet, um die spottenden ‘warum` des Egos zufrieden zu stellen. Das Ausmass dieses Selbstbetruges ist so bedeutend, dass es den philosophischen Rationalismus zur Farce machen könnte» (CP 1.631, Hervorh. des Autors). Dieser Text scheint auf das Unbewusste zu zielen, nicht auf das Vorbewusste. Freud zeigt auf: «Unseren Begriff des Unbewussten gewinnen wir also aus der Lehre von der Verdrängung» (1982c, S. 284). Die Sorte Selbstbetrug, auf die Peirce sich hier bezieht, scheint jener Art Mechanismus eng verwandt zu sein, bei dem gemäss Freud Vorstellungen, Bilder, Wünsche und Vorfälle verdrängt werden. Kurz, es scheint sich um die Vorstellung vom Unbewussten im eigentlichen Sinne zu handeln.

Der andere der eben zitierten Texte, in welchem Peirce das menschliche Bewusstsein metaphorisch als abgrundtiefen See beschreibt, fährt jedoch in einer Weise fort, die in eine andere Richtung weist. Der Autor baut seine Trope aus, indem er nahe legt, dass sich in den Wassern des Sees «unzählige Objekte in verschiedenen Tiefen befinden. Gewisse Kräfte geben gewissen Arten dieser Objekte einen Aufwärtsimpuls, der eventuell stark genug ist und lange genug anhält, um sie in die oberen Schichten zu bringen, wo sie sichtbar werden» (CP 7.547). Da gibt es also einerseits eine überall präsente Kraft, die alle mentalen Objekte nach unten zieht, so dass sie gezwungen werden, in Vergessenheit zu versinken. Dann gibt es aber auch eine selektive Aufwärtskraft, die auf einige der Objekte wirkt (sie manchmal vielleicht auch anlockt) und sie wieder an die Oberfläche des Bewusstsein schiebt. Es sieht nun so aus, als ob das, was nicht bewusst ist, höchstens vorbe- 
wusst im Freud'schen Sinn ist, nämlich ausserhalb des Bereichs der unmittelbaren Aufmerksamkeit, aber immer leicht zu erreichen. Es ist nicht das Unbewusste der Psychoanalyse, das durch Widerstand gegenüber bewusstem Ausdruck in unverkleideter Form gekennzeichnet ist. Doch der Schein trügt, weil Peirce’ Blick Platz macht für das Unbewusste auch in einem strikt Freud'schen Sinne.

Zusätzlich zur allpräsenten Abwärts-Kraft oder einer Menge von Kräften ${ }^{13}$ (beachten Sie z. B. seine Bemerkungen zu Ermüdungserscheinungen [Fatigue] [CP 1.390; 7.552: MS 318: MS 1107]) könnte Peirce wohl widerspruchsfrei und zwanglos die Präsenz anderer, selektiverer Kräfte (wie z. B. die der Verdrängung oder der Zensur im Freud'schen Sinne) akzeptieren. Der oben zitierte Text (CP 1.631) legt sogar nahe, dass sich Peirce dieser selektiven Kräfte ziemlich genau bewusst war. Peirce' Aussagen zum Unbewussten betreffen also mehr als nur das Vorbewusste.

\section{Das Vorbewusste: Charakteristische Aufmerksamkeit}

\section{kompetenter Akteure}

Das Vorbewusste verdient es indessen auch nicht, einfach ignoriert zu werden, vor allem deshalb nicht, weil Peirce die Gewohnheiten betonte und nachdrücklich darauf insistierte, dass das glatte Funktionieren von Gewohnheiten normalerweise mit einer minimalen Aufmerksamkeit einhergehe. So schenken wir zum Beispiel, während wir die Türe öffnen, der Türklinke kaum Beachtung, ausser wenn sie sich, entgegen unseren Erwartungen, unseren tief eingeprägten und fein koordinierten Bewegungen der Hand, des Armes und der Augen widersetzt. Die Effektivität und Geschwindigkeit, mit der wir unsere bescheidenen Zwecke verfolgen, zeigt, dass es sich bei der menschlichen Semiose um einen typisch vorbewussten Prozess handelt. Dies ist bemerkenswert, denn die Verfolgung dieser Ziele hängt ab von einer sofortigen und leichtfüssigen Interpretation der wahrhaft kaleidoskopischen Muster unvermittelt aufleuchtender Signale. Wir sind uns dieser Gewandtheit normalerweise nicht bewusst, können uns aber der Türknöpfe, die wir drehen, der Tasten, die wir betätigen, der Bändel, die wir schnüren ohne weiteres bewusst werden.

Diese einfache Beobachtung veranlasst mich, etwas vorzuschlagen, was manchen vielleicht als kühne Hypothese erscheint. Wir brauchen nicht nur eine Theorie, die erklärt, wie Ideen im Unbewussten wirksam sein können, obwohl sie für das Bewusste vollkommen unerreichbar sind (also wie Vorstellungen verdrängt sein können), sondern es muss auch erklärt werden, wie vorbewusste Ideen bewusst werden. Angesichts der Effektivität und Flexibilität der Mehrzahl unserer Gewohnheiten würde ich sagen, dass die meisten unserer Vorstellungen vorbewusst 
sind. Semiotisch gesprochen ist der Hauptteil der Zeichen, die wir interpretieren, nicht im Fokus unserer erhöhten Aufmerksamkeit; und in noch vermehrtem Masse trifft dies für den Prozess zu, mittels dessen wir die Zeichen interpretieren. Kleine Störungen, die unvermeidbar jegliches durchzuführende Vorhaben akzentuieren, laden uns ein, aufmerksamer zu sein als gerade bevor wir den Widerstand spürten; sie bedeuten die Abwesenheit einer perfekten Abstimmung zwischen den Gewohnheiten, die unserer verkörperten Handlungskraft zugehören und denjenigen, die Bestandteile der Objekte sind, denen wir begegnen. Zudem ist die Bandbreite motivierender Handlungsziele, ${ }^{14}$ die menschliche Unternehmen anstossen, unterhalten und modifizieren und - selbstverständlich - oft auch veranlassen, sie aufzugeben, so gross, dass auch Gewohnheiten geprägt wurden, die unsere Aufmerksamkeit steigern. So hat z. B. der Musiker, der durch den Wald spaziert, vielleicht die Gewohnheit, musikalische Qualitäten in den zufälligen Tönen zu hören, die von verschiedenen Richtungen auf ihn einströmen. Ich glaube, dass diese beiden Faktoren - das Fehlen der perfekten Abstimmung und die gewohnheitsmässige Aufmerksamkeit - weitgehend erklären, wie sich die vorbewussten Vorstellungen in Richtung Bewusstsein bewegen, obwohl die Mehrheit von ihnen nicht wirklich auf sich aufmerksam machen kann. Vielleicht sind wir in den meisten Tagesangelegenheiten sehr viel traumwandlerischer als wir denken. Aber auch so erfahren wir im Laufe unserer Routine viel öfter leichte, unbestimmbare Irritationen als ein plötzliches Aufschrecken. Diese Irritationen und unsere Aufmerksamkeit erfordernden Gewohnheiten arbeiten zusammen als Aufwärts-Impulse; sie betätigen sich als selektive Kräfte, die einige Objekte an die sichtbare Oberfläche des abgrundtiefen Sees treiben, der Bewusstsein genannt wird. Diese Objekte sind meistens unter, oft gerade knapp unter der Oberfläche des Bewusstseins.

Die Beweglichkeit, die als Resultat der fein nuancierten und flexiblen Abstimmung erlebt wird, welche uns unsere verkörperte Kraft in ungezählten Gelegenheiten ermöglicht, könnte als Firstness der Thirdness bezeichnet werden. Es handelt sich um den qualitativen Aspekt einer funktionierenden Gewohnheit, der sich anfühlt wie die qualitative Vollständigkeit einer Melodie, in welcher eine Phrase mit der anderen verschmilzt. Die plötzliche Unterbrechung einer Gewohnheit ist schmerzhaft (obwohl, je nach Umständen, vielleicht nur ein wenig); eine solche Erfahrung als schmerzhaft zu charakterisieren bedeutet für Peirce, dass sie gezeichnet ist durch den Kampf in Richtung Ruhezustand ${ }^{15}$ (CP 5.113). Im Gegensatz dazu ist die flüssige Ausübung einer Gewohnheit angenehm, der Genuss liegt «in einer besonderen Art des Bewusstseins, das zum Bewusstsein gehört, eine 
Abstraktion vorzunehmen, in dem nicht das Gefühl, sondern eher die Erkenntnis hauptsächlicher Bestandteil ist» (CP 5.113). Das heisst, weder Schmerz noch Genuss sind Phänomene, bei denen die Firstness im Vordergrund steht. Schmerz ist in erster Linie Second, während Lust prinzipiell zum Third gehört. Die Lust als Eigenschaft der Thirdness ist solcherart, dass das inhärente Vergnügen der kunstvollen Ausführung, unabhängig davon wie rudimentär oder instinktiv auch immer derVorgang sei, die Ausführung selber antreibt und führt; es ist eine Thirdness, die ihre Eigendynamik mit der Zufälligkeit der Umstände vereint.

Die feine Abstimmung zwischen inhärenter Dynamik und Umgebung kann so gründlich sein, dass Secondness nur noch als Antrieb spürbar ist, der zu immer befriedigenderen Vermittlungen einlädt (ähnlich wie Dissonanzen in einer musikalischen Komposition im Dienst einer noch melodischeren Auflösung stehen). Das Second erleichtert dann den Fluss des Third, so dass die entscheidende Eigenschaft - seine rohe Andersheit - ausgelöscht wird. Dabei kann die Flüssigkeit der Gewohnheit sich bis zu einem solchen Grad als Firstness der Thirdness erweisen, dass sie dem Primisense ${ }^{16}$ gleicht, diesem Modus des Bewusstseins, in welchem qualitative Unmittelbarkeit und ungebrochene Integrität sich von Altersense und Medisense abheben (CP 7.551).

Primisense oder Bewusstsein als Firstness - als pure Spontaneität, undifferenziertes So-Sein, absolute Unbeschreiblichkeit usw. - ist so verschieden von dem, wie körperliche und weltliche Vorgänge und Objekte gewöhnlich beachtet werden (was wir üblicherweise Bewusstsein nennen), dass man mit Peirce sagen könnte, was er selbst zu James bezüglich dessen «reiner Erfahrung» sagte: «Was $\mathrm{Du}$ «reine Erfahrung` nennst, wird überhaupt nicht erfahren und sollte unbedingt einen eigenen Namen haben. Es ist ausgesprochen unmoralisch, Wörter so zu missbrauchen, denn es hindert die Philosophie daran, eine Wissenschaft zu werden» (CP 7.301). Aber eine so scharfe Entgegnung wäre dann doch nicht angemessen, denn in Wahrheit gibt Peirce sich grosse Mühe (unter anderem in mehreren Briefen an James) zu zeigen, dass die Gleichsetzung von Bewusstsein mit Gefühl gut begründet ist, und dass zusätzlich zumindest eine der möglichen Bedeutungen von «Gefühl» den Peirce’schen Gebrauch stützt. Mit dem Erscheinen von uns allen und vermutlich auch anderer uns ähnlicher Organismen platzt die Welt in die Szene, aus einem Chaos unpersönlichen Fühlens heraus - und dies genau in der Weise, wie sie Peirce in der Schlusssequenz von «The Architecture of Theories» beschreibt (CP 6.33) - nur um sich einer bereits bestehenden Welt (wenn auch einer Welt, die sich in Evolution befindet) entgegenzusetzen. Es ist nicht so, dass Ideen-Zeichen (idea-signs) zuerst bewusst wären und dann durch eine allgegenwärtige oder eine 
spezifische Kraft aus dem Bewusstsein verbannt würden; eher schon ist es so, dass ursprünglich solche Zeichen nur in einer sehr schwachen Form bewusst sind (das heisst als Primisense) (CP 7.551). Sie müssen auf die Stufe dessen gebracht werden, was wir normalerweise unter Bewusstsein verstehen; einem Sinn für Achtsamkeit in der beides, der Sinn für Andersheit und jener für die Bedeutung des Objekts, unmittelbar Bestandteil sind.

Unser alltägliches Bewusstsein ist nämlich das Resultat eines Schwankens zwischen flüssig funktionierenden Gewohnheiten, innerhalb derer Andersheit und Bedeutung der Objekte und Ereignisse mehr oder weniger wirkungslos bleiben einerseits, und plötzlichem Abstoppen unserer Bemühungen andererseits, bei welchem Andersheit und Bedeutung auffallen. Anders gesagt, es bewegt sich abwechslungsweise zwischen Phasen der Firstness der Thirdness (ein vollkommen ungestörtes In-Szene-Setzen der Absicht) einerseits und jenen von Thirdness andererseits, in der Secondness und Thirdness sich vordrängeln und versuchen, das jeweils andere zu dominieren. Absichtsvolles Handeln, der Grundstoff unserer Alltagserfahrung, ist wie ein gewobener Teppich, in dem sich Erfüllung und Frustration, gewandte Ausführung und blindes Straucheln ineinander flechten.

Erfahrungen von ausgeprägter Secondness (z. B. wenn man sich die Zehe anschlägt) sind gewaltsame Ausrufezeichen, die zuerst einmal unmittelbares Handeln verlangen (oft indem wir einfach unsere Tätigkeit unterbrechen, im Falle der angeschlagenen Zehe von einem Fuss auf den anderen hüpfen und unser Schicksal verfluchen). Reflexive Personen werden eingeladen, das eigene Verhalten zu überdenken. Im Laufe dieser Überlegungen verwandelt sich das Ausrufezeichen in eine Reihe von Fragezeichen. Weiss ich, was ich tue? Weiss ich, wo ich bin? In was für einem Kontext versuche ich, meine Absichten zu verfolgen? Ist für meine eigene Sicherheit angemessen gesorgt? Aber ich wiederhole: Das übliche Alltagsbewusstsein ist zum Grossteil nicht eine Serie aufrüttelnder Frustrationen. Es ist eher ein gleitendes Feld, bei dem hier das Gefühl eines ungehinderten Ablaufes vorherrschend ist, da eine schwierige Balance zwischen widerständigen Kräften und erleichternden Umständen zu halten ist (charakteristischerweise zwischen einem Altersense im Verhältnis zu zweckgerichtetem Verhalten und einem Medisense, der der interpretierenden Erfindungsgabe entspricht).

Bei Peirce sind menschliche Wesen ziemlich kompetente Akteure, und das Alltagsbewusstsein, das ich oben beschrieben habe, ist das praktische Bewusstsein kompetenter Akteure (vgl. Giddens, 1991; Colapietro, 1995). Dies über menschliche Wesen zu sagen, bedeutet implizit, dass wir bis zu einem gewissen Grade unwissende Tölpel sind. Aber das Ausmass, in dem wir Spielzeug von Kräften jenseits 
unseres Bewusstseins und unserer Kontrolle sind, sollte auch nicht übertrieben werden. Angesichts Peirce' Bekenntnis zur Praxis (vgl. Colapietro, 1991, 141-143; 152-155) konnte er das Bewusstsein unmöglich nur als Epiphänomen sehen. Während bewusstes Handeln tatsächlich nur eine bescheidene Rolle spielt in der Bestimmung des gegenwärtigen Verhaltens, und vielleicht bei der Bildung von Gewohnheiten sogar noch weniger Einfluss hat, ist das Bewusstsein dafür, wie wir handeln wollen, und in seinem Einfluss darauf, wie wir eingestellt sind, sehr wichtig. Aber das Bewusstsein wird daran gehindert, bei der oft prekären Bildung von «normalen Interpretanten» mitzuwirken (Savan, 1987/88, p. 61-62). Die mehr oder weniger gesetzmässigen Wege, die die Evolution solcher Interpretanten behindern, sind, gemäss meiner Deutung, die Mechanismen derVerdrängung, die Strategien, mit denen das Unbewusste die Fähigkeit des Selbst, besser angepasste, angemessen flexible und wirkungsvoll fein abgestimmte Wahrnehnmungsmuster, Affekte und Willensäusserungen zu entwickeln, zum Erliegen bringt.

Wir haben oben bemerkt, dass das Ausmass an Selbsttäuschung derart ist, «dass es die rationale Philosophie zur Farce macht» (CP 1.631). Im Gegensatz zum philosophischen Rationalismus verteidigt Peirce aber explizit den philosophischen Sentimentalismus. Diese Unterstützung basiert auf seiner Überzeugung, dass die Gefühle die Substanz unserer Seele ausmachen, während Erkenntnis [cognition] nur Oberfläche ist. Bewusste Vernunft, der Ort, wo Erkenntnisse formal artikuliert und analysiert, sowie Schlussfolgerungen gezogen werden, ist nur ein oberflächlicher Teil der menschlichen Psyche. Es ist sogar so, dass die Vernunft «an das Gefühl als letzte Instanz appelliert. Das Gefühl seinerseits fühlt sich selber als der Mann [oder die Frau, an den der Appell sich richtet, Anm. V.C.]. Das ist meine einfache Legitimation des philosophischen Sentimentalismus» (CP 1.632). Peirce zögert im zitierten Text nicht, Gefühl und Instinkt im selben Atemzug zu nennen (vgl. z.B. CP 1.628). Er beteuert auch: «Instinkte können gebildet werden und wachsen» (CP 1.648). Aber das Wachstum von Instinkt und Gefühl findet nur durch einen langsamen Prozess weitgehend unbewusster Versickerung statt, mit dem Resultat, dass das Wachstum «nach und nach den wirklichen Kern des Wesens» (CP 1.648) erreicht. So wie ich das verstehe, ist dieser Kern, der sich solchermassen formt, ein Bündel von Gewohnheiten der Zu- und Abneigung, von Sympathie und Antipathie - es handelt sich um die Gefühlsgewohnheiten, die letztlich den andern Gewohnheiten zugrunde liegen, und zwar beiden, sowohl jenen mit nach aussen gerichteter Ausführung wie auch dem Imaginativen, Reflexiven (d.h. Handlung und Denken). 
Ich fasse zusammen: Geist ist gleichzeitig weiter und tiefer als Bewusstsein. Nach Peirce gibt es drei Formen des Bewusstseins: Gefühl der qualitativen Unmittelbarkeit, Erfahrung brutaler Gegenkräfte und das Gewahr-Werden der kontinuierlichen Vermittlung. Eine der besten Erläuterungen dieser Formen ist noch immer Nathan Housers Artikel «Peirce’s General Taxonomy of Consciousness», der 1983 in den «Transactions of the Charles S. Peirce Society» publiziert wurde. Denn was wir gewohnt sind, als Alltagsbewusstsein zu bezeichnen, ist in Wirklichkeit eine komplexe Sache, in welcher die Firstness des Gefühls, die Secondness der Opposition und die Thirdness der Mediation unausgesetzt verwoben werden und gleitende Muster kontinuierlicher Veränderung bilden. Das charakteristische Niveau des Bewusstseins, in welchem kompetente Akteure sich bewegen und leben, entspricht im strengen Sinne dem Vorbewussten. Aber unsere Absichten und Vorhaben werden natürlich nicht nur durch nicht vorhersehbare Umwelthindernisse gebremst, sondern ebenso durch nicht eingestandene psychische Bürden. Wir können ebenso mit uns selber in Konflikt sein, wie mit unserer Umwelt. Dies spricht dafür, das Unbewusste anzuerkennen. Analog zu Peirce' Analyse des Bewusstseins in drei Modi (und speziell angesichts seiner Triadomanie! [CP 1.568]), scheint es mir plausibel, dass er auch für das Unbewusste drei Aspekte annehmen würde.

\section{Das Unbewusste im Licht der Peirce'schen Kategorien}

Wie oben angeführt, kann ein Abrutschen in theoretische Schwäche und leichtfertigen Eklektizismus nur abgewendet werden durch ein beständiges Vertrauen in Peirce' kategoriales Schema. Positiv formuliert ermächtigt uns dieses Vertrauen in Peirce' Kategorien, die formalen und dynamischen Beziehungen zu verfolgen, die ausschlaggebend sind für die erfolgreiche Ausführung unseres theoretischen Vorhabens. Darüber hinaus rüstet uns allein die kategoriale Analyse für eine Erkundung des Unbewussten unter wahrhaft peirceanischer Perspektive; denn obwohl das Unbewusste eindeutig einer semiotischen Interpretation bedarf, bedingt die semiotische Deutung ihrerseits kategoriale Analysen auf verschiedenen Abstraktionsniveaus, die von der Spezialebene der Phänomenologie selber bis zur allgemeinen ${ }^{17}$ Ebene der Experimentalpsychologie reichen. In den Worten von David Savan: «Peirce’ drei Kategorien spielen eine wichtige Rolle in jedem Aspekt seines Denkens, für seine Semiotik indessen sind sie absolut entscheidend. Peirce' erste publizierte Arbeit über die Kategorien stellte diese einfach als den grundlegendsten Teil seiner Theorie der Zeichen dar. Semeiotik ${ }^{18}$ in all ihren Definitionen, Unterteilungen, Trichotomien, Zweigen und Kombinationen ist gemäss Peirce vollständig bestimmt durch die kategoriale Theorie» (1987/88, 15; vgl. auch Shapiro, 
1983). Demgemäss wäre nun der Entwurf einer kategorialen Beschreibung des Unbewussten angebracht.

Wollheim schlägt ja vor, Freuds Unbewusstes als zweiteilig anzusehen: «Zum Schluss zerfällt das Unbewusste in zwei Teile, in das «ursprünglich Mitgebrachte` und in das während «der Ichentwicklung Erworbene $»^{19}$ (Wollheim, 1972, S. 144; Freud, 1938, XVII, S. 84f.). Demgegenüber fordern Peirce' Kategorien uns auf, konzeptuelle Möglichkeiten auszuprobieren, gemäss denen das Unbewusste in drei Teile aufzuteilen sei. Aber was könnten diese sein? Erlauben Sie mir versuchsweise das Folgende vorzuschlagen: Das Unbewusste selber ist - irgendwie paradox - gemäss Peirce'scher Terminologie bereits ein Phänomen. Denn so wie alles zumindest potentiell ein Zeichen ist, kann gleichzeitig auch alles ein Phänomen sein. «Das, was einem Phänomen zu Grunde liegt und es bestimmt, ist also in gewissem Sinne ein Phänomen» (CP 7.569). Die unbewussten Inhalte als das, was den unmittelbar oder leicht erreichbaren Regionen des psychischen Lebens zu Grunde liegt und sie bestimmt, verbergen sich hinter Masken und geben sich zur gleichen Zeit mit ihren komplexen Verkleidungen und Täuschungen zu erkennen. Kurz gesagt, es ist «selbst in einem Mass ein Phänomen»- etwas, das in einer gewissen Art und Weise und in einem bestimmen Kontext erscheint, wenn denn die Beobachter erfahrene Interpreten sind (vgl. CP 1.35), und wenn wiederum die Interpreten Theoretiker der Kategorien sind. Natürlich kann man einwerfen, dass diese Interpreten und Theoretiker das Phänomen, das sie angeblich beobachten, offensichtlich erfinden; aber dieser Einwand könnte nur aufrechterhalten werden, wenn angenommen wird, dass interpretative und theoretische Schemen überhaupt keine Rolle dafür spielen, was in einem gegebenen Kontext als Phänomen gelten soll (Short, 1988). Diese Annahme ist jedoch unhaltbar.

Wenn das Unbewusste als Phänomen gelten soll, auch wenn dies nur in einem schwachen und tatsächlich problematischen Sinne zutrifft, muss es in einem ihm eigenen Sinne die unabdingbaren Merkmale eines Phänomens aufweisen. Und - ich wiederhole - was mag das sein? Einmal wäre das Unbewusste als Phänomen, in welchem die Firstness vorherrscht, Geist in schrankenloser Spontaneität, unausrottbarer Mutwille, und unerschöpfliche Fruchtbarkeit; (vor allem) ikonische Ressource und symbolisches Potential. Zweitens, das Unbewusste als das Andere, als Second, wäre das, was dem Bewusstsein entgegensteht, seine unbezähmbare Spontaneität, die zielgerichtet als störende Kraft arbeitet, als anarchistischer Impuls. ${ }^{20}$ Aber brutale Tyrannei ist eine vollständig destruktive Sache, die ihre eigene Vernichtung in sich trägt. Die verstohlene Tyrannei nicht akzeptierter Symbole und die unterdrückten Erzählstrukturen dienen der Hegemonie 
des Unbewussten weit besser. Jedoch in dem Augenblick, indem wir Symbole und Erzählstrukturen einführen, wird das Unbewusste zum Phänomen, in dem die Thirdness vorherrscht (denn die geheime Thirdness ist eine sogar noch komplexere Form der Mediation als die gradlinige Symbolisierung, insofern sie von der Symbolisierung abhängig, aber nicht auf sie reduzierbar ist). Mit anderen Worten, wir charakterisieren das Unbewusste selber als das, was vermittelt zwischen der vorbewussten Handlungsautorschaft und den wechselnden Schauplätze unserer verschiedenen Tätigkeiten. Oft, wenn nicht typischerweise (vgl. Dewey, 1925, S. 227-228; 239), ${ }^{21}$ vermittelt das Unbewusste unsere Reaktionsweisen solcherart, dass sie infantil erscheinen - oder, zumindest, alles andere als subtil und nuanciert. Merke: Das Unbewusste auf diese Weise konzipiert befindet sich eher zwischen uns und unserer Welt; nicht hinter oder unterhalb der bewussten Region unseres psychischen Lebens.

\section{Schlussfolgerung}

Von einer detaillierten kategorialen Beschreibung des Unbewussten ausgehend (natürlich einer detaillierteren als meiner viel zu flüchtigen Skizze) sollten wir in Richtung einer ebenso genauen semiotischen Beschreibung fortschreiten, in der Gewohnheiten als Interpretanten eine, wenn nicht die zentrale Rolle spielen würden (de Lauretis, 1984; 1994). Aber ach, was unser Vorhaben gleichzeitig so berauschend und frustrierend macht, ist der geforderte gewaltige Zeit- und Energieaufwand, nur um Peirce einzuholen, wo er, speziell in der letzten Dekade seines Lebens, schon war! Das bedeutet praktisch, dass wir das Unbewusste (neben anderen Themen) fortwährend neu denken müssen, während wir darüber nachdenken. Auch wenn wir, wofür Teresa de Lauretis in ihrem neusten Buch argumentiert, Freud mit Peirce lesen sollten (1994, Kapitel 7; vgl. 1984, Kapitel 6), müssen wir auch Peirce mit Freud lesen - und zweifellos mit Jung, Lacan, Kristeva und anderen. Wir sollten das Unbewusste im Licht der peirceanischen Kategorien und Semiotik lesen, ebenso wie den Mann und Philosophen Peirce im Licht der psychoanalytischen Theorien (vgl. Colapietro, 1995). Meine bescheidene Hoffnung ist, dass ich, indem ich diese Notizen für die Skizze einer peirceanischen Theorie des Unbewussten zusammengestellt habe, nicht nur einige Anregungen plausibler gemacht habe, sondern auch hilfreiche Tipps dafür gebe, wie eine so aufregende, aber auch anstrengende Aufgabe angegangen werden könnte. 


\section{Literatur}

Braga, L. S. (1993). Difficulties and strategies in applying Peirce's semiotics. Semiotica, 97(3/4), 401-410.

Colapietro, V. (1995). Philosophical biography: Preliminary reflections on a distinctive form of historical inquiry. Corrington, R. \& Deely, J. (Eds.), Semiotics 1993 (S. 583-589). NY: Peter Lang.

Colapietro, V. (1992). Purpose, power and agency. The Monist, 75(4), 423-444.

Colapietro, V. (1991). Two rival conceptions of the semiological ideal: Peirce versus Saussure. Face, Revista de Semiotica e Comunicacao, numero especial, 135-158.

Colapietro, V. (1989). Peirce's approach to the self: A semiotic perspective on human subjectivity. Albany: SUNY Press.

de Lauretis, T. (1994). The practice of love: Lesbian sexuality and perverse desire. Bloomington: Indiana University Press.

de Lauretis, T. ( 1984). Alice doesn't: Feminism, semiotics, Cinema. Bloomington: Indiana University Press.

Dewey J. (2004). Menschliche Natur, ihr Wesen und ihr Verhalten. Zürich: Pestalozzianum Verlag.

Englisch: Human nature and conduct. Middle Works MW 14.

Dewey, J. (1996). Die Öffentlichkeit und ihre Probleme. Bodenheim: Philo Verlagsgesellschaft.

Dewey J. (1995). Erfahrung und Natur. Frankfurt a. M.: Suhrkamp.

Englisch: Experience and nature (1925).

Eco, U. (1976). A theory of semiotics. Bloomington: Indiana University Press.

Deutsch: (1972). Einführung in die Semiotik. München: W. Fink.

Fisch, M. H. (1986). Peirce, semeiotic, and pragmatism. Bloomington: Indiana University Press.

Freud, S. (1982a/1915). Das Unbewusste. Studienausgabe Bd I. (S. 121-173). Frankfurt a. M.: Fischer.

Freud, S. (1982b/1915-17). Vorlesungen zur Einführung in die Psychoanalyse. Studienausgabe Bd. I. (S. 34-445). Frankfurt a. M.: Fischer.

Freud, S. (1982c/1923). Das Ich und das Es. Studienausgabe Bd III. (S. 273-330). Frankfurt a. M.: Fischer.

Freud, S. (1982d/1930). Das Unbehagen in der Kultur. Studienausgabe Bd IX. (S. 191-270). Frankfurt a. M.: Fischer.

Freud, S. (1938). 4. Kapitel Psychische Qualitäten. Gesammelte Werke Band XVII (S. 79-86). Frankfurt a. M: Fischer Taschenbuchverlag. 1999. 
Gardner, S. (1993). Irrationality and the philosophy of psychoanalysis. Cambridge: Cambridge University Press.

Giddens, A. (1991). Modernity and self-identity. Stanford, CA: Stanford University Press.

Hardwick, Ch.S. (1977). Semiotic and significs: The correspondence between Charles S. Peirce and Victoria Lady Welby. Bloomington: Indiana University Press.

Houser, N. (1992). Introduction. Houser, N. (Ed.), The essential Peirce, selected philosophical writings. Vol. 1. (S. IXX-XLI). Bloomington \& Indianapolis: Indiana University Press.

Houser, N. (1983). Peirce's general taxonomy of consciousness. Transactions of the Charles S. Peirce Society, XIX, S. 331-359.

Lacan, J. (1977). Ecrits: A selection. Translated by Alan Sheridan. NY: W.W. Norton \& Company.

McDermott, J. J. (1976). The culture of experience. NY: NYU Press.

Peirce, C.S. (1995). Religionsphilosophische Schriften. Übersetzt, eingeleitet, kommentiert und herausgegeben von H. Deuser. Hamburg: Felix Meiner Verlag.

Peirce, C. S. (1970). Schriften II, Vom Pragmatismus zum Pragmatizismus. Apel, K. O. (Hrsg.). Frankfurt a. M.: Suhrkamp.

Peirce, C. S. (1958). The Collected Papers of Charles Sanders Peirce VII-VIII. A.W. Burks (Ed.). Cambridge, MA: Harvard University Press. (Zitiert als CP, gefolgt von Bandnummer. Paragraph).

Peirce, C.S. (1931-1935). The Collected Papers of Charles Sanders Peirce I-VI. Hartshorne, Ch. \&Weiss, P. (eds.). Cambridge, MA: Harvard University Press.

Peirce, C. S. Unveröffentlichte Schriften werden als MS, mit der Manuskriptnummer nach R.S. Robin zitiert. Robin, R.S. (1967). Annotated Catalogue of the Papers of Charles S. Peirce, Amherst: The University of Massachusetts Press.

Pharies, D.A. (1985). Charles S. Peirce and the linguistic sign. Philadelphia: Benjamins North America.

Ricoeur, P. (1970). Freud and philosophy: An essay on interpretation, translated by Denis Savage. New Haven: Yale University Press.

Savan, D. (1987/88). An introduction of C. S. Peirce's full system of semeiotic. Toronto: Toronto Semiotic Circle.

Shapiro, M. (1983). The sense of grammar: Language as semeiotic. Bloomington: Indiana University Press.

Short, T. L. (1988). The growth of symbols. Cruzeiro Semiotico, 8, 81-87. 
Wilshire, B. (1993). Body-Mind and subconsciousness: Tragedy in Dewey's life and work. Stuhr, J. J. (Ed.), Philosophy and the reconstruction of culture. (pp. 257-272). Albany: SUNY Press.

Wollheim, R. (1972). Sigmund Freud. München: dtv.

Englisch: (1971): Sigmund Freud. NewYork: Press Syndicate of the University of Cambridge.

\section{Anmerkungen}

1 Der Artikel «Notes for a Sketch of a Peircean Theory of the Unconscious» erschien 1995 in den «Transactions of the Charles S. Peirce Society». Vol. XXXI: 3, p. 482-506. Die Rechte für Übersetzung und Publikation wurden uns freundlicherweise von der Indiana University Press zur Verfügung gestellt.

2 Diese Arbeit war das Resultat eines Vortrages am fünften Kongress der International Association for Semiotic Studies - Association Internationale de Sémiotique (IASS-AIS) an der University of California, Berkeley, 13. Juni 1994. Ich bedanke mich bei meinen Kollegen und Kolleginnen, die mit wertvollen Kommentaren zu früheren Entwürfen dieser Arbeit zum Gelingen beigetragen haben, insbesondere sind dies Robert Ashmore, Anne Freadman, Josephine Glorie, Wojciech Kalaga, Elisabeth Saporiti und Kory Sorrell.

3 Der Ausdruck habit ist einer der zentralsten Begriffe im Peirce'schen Universum. Ich übersetze ihn hier durchwegs mit Gewohnheit. Anm. der Übersetzerin.

$4 \quad$ Für Peirce' Kategorien von First, Second und Third, vgl. die Artikel von Saller und Barwinski in diesem Heft. Anm. der Übersetzerin.

5 Ich entnehme die deutsche Fassung des Zitates bis zum Strichpunkt der deutschen Übersetzung (1972), der Nachsatz fehlt im deutschen Exemplar. Anm. der Übersetzerin.

$6 \quad$ Colapietro benützt das Wort signficant und betont das Vorhandensein des Zeichenhaften im Wort selber mit folgender Schreibweise: sign-ificant. Anm. der Übersetzerin.

7 Die Übersetzung der englischen Zitate stammt von Vera Saller. In Werken, die bereits in Deutsch publiziert wurden, habe ich wenn immer möglich aus dem deutschen Werk zitiert. Anm. der Übersetzerin.

8 Colapietro benützt hier das englische Wort purpose, das bei Peirce einen sehr umfassenden Sinn hat. Nathan Houser vergleicht den Peirce'schen purpose mit Brentanos Intentionalität (1992, S. XXXV). Im Gegensatz zu jener ist der purpose aber nicht nur im menschlichen Geist vorhanden, sondern teleologische Gesetze zeichnen die ganze Evolution aus. Anm. der Übersetzerin.

$9 \quad$ Vgl. Anm. 6.

10 Im Original dis-ease, un-wohl. Anm. der Übersetzerin.

11 Mein Dank gilt Robert Ashmore, der mir bei der Entwicklung dieser Gedanken behilflich war. Zur Art und Weise, wie Gewohnheiten Aufmerksamkeit und Bewusstsein anregen oder erleichtern können ist Dewey (2004) speziell erhellend. Sehr viel mehr als James und sogar stärker als Peirce spricht er sich dafür aus, dass diese Möglichkeit einigen Typen von Gewohnheitsbildung inhärent sei.

12 Colapietro verwendet hier das Wort idioscopic; im zweiten Teil des Satzes cenoscopic. Das Wortpaar übernimmt Peirce von Jeremy Bentham. Der finnische «Commens Peirce Dictionary» erläutert dieses Wortpaar mit dem folgenden Peirce-Zitat. "The sort of science that is founded upon the common experience of all men was recognized by Jeremy Bentham under the name of cenoscopy, in opposition to idioscopy, which discovers new phenomena" 
(CP 8.199, 1905) http://www.helsinki.fi/science/commens/terms/idioscopy.html (Letzter Aufruf 31.1.2014), Anm. der Übesetzerin.

13 In einer ausführlicheren Behandlung von Peirce' Verständnis des Unbewussten müsste die Deutung unseres geistigen Lebens als Austausch von Kräften und die dahinter stehende Konzeption von Kraft näher untersucht werden. Dabei bin ich der Meinung, dass es lehrreich ist, Ähnlichkeiten und Unterschiede zwischen ihm und Freud herauszuarbeiten. Im Speziellen ist die Frage zentral, inwieweit und in welcher Art das Freud'sche theoretische Festhalten an einem durchgehenden Determinismus und an der menschlichen Psyche als homöostatischem System seine eigene hermeneutische (und damit semiotische) Annäherung unterwandert (vgl. Ricoeur, 1970).

In diesem Zusammenhang ist es erhellend, Deweys Beobachtungen in «Die Öffentlichkeit und ihre Probleme» in Erinnerung zu rufen: «Verbundene Tätigkeit findet unter menschlichen Wesen statt; wenn aber nichts sonst stattfindet, geht sie so unvermeidlich in eine andere Form wechselseitig verbundener Tätigkeit über, wie das Zusammenspiel von Eisen und dem Sauerstoff des Wassers. Was dabei geschieht [und wir könnten anfügen: innerhalb genauso wie zwischen menschlichen Wesen, Anm. V.C.], ist ganz in Begriffen von Energie oder, wie wir im Falle menschlicher Interaktion sagen, von Kräften [force] beschreibbar» (Dewey, 1996, S. 131). Aber das ist natürlich nicht das Ende der Geschichte, denn Zeichen und Symbole verändern das Zusammenspiel von intra- und interpersonellen Kräften (sogar wenn wir annehmen, dass diese Kräfte selber immer schon zu einem gewissen Grad und in einer gewissen Weise semiotische Phänomene sind). «Nur wenn dabei Zeichen oder Symbole von Tätigkeiten und ihren Resultaten vorhanden sind, kann der Fluss von aussen betrachtet, zwecks Prüfung und Begutachtung aufgehalten und dann reguliert werden. Der Blitz trifft und spaltet einen Baum oder einen Stein und die entstehenden Stücke nehmen den Prozess derWechselwirkung auf und setzen ihn fort, und immer so weiter. Wenn aber die Phasen des Prozesses durch Zeichen [und Symbole, denn sie zeigen sich immer für menschliche Zeugen und Teilnehmer, Anm. V.C.] dargestellt werden, ist ein neues Medium dazwischengetreten. Wenn Symbole miteinander verbunden werden, werden die wichtigsten Beziehungen eines Ereignisverlaufes aufgezeichnet und als Bedeutungen bewahrt. Erinnerung und Voraussicht sind möglich [...]» (Dewey ebd., 131; 132). «Eine Gemeinschaft stellt folglich eine Ordnung von Energien dar, die in eine Ordnung von Bedeutungen umgewandelt ist, welche von all jenen, die mit der verbundenen Tätigkeit befasst sind, geschätzt werden und auf welche diese untereinander Bezug nehmen» (ebd.). So ist auch das Selbst eine fortwährende Verwandlung eines Kräfteverhältnisses in Symbole. Deshalb ist, «Kraft [...] dadurch nicht abgeschafft, vielmehr wird sie in ihrem Gebrauch und ihrer Ausrichtung durch Ideen und Gesinnungen, die mittels Symbolen ermöglicht werden, verwandelt» (ebd.). Was Dewey hier unterstreicht, ist ein semiotischer Zugang ähnlich jenem von Peirce und ein bisschen anders als Freud (weil Freuds mechanistische und hermeneutische Seiten angesichts seiner theoretischen Verbindlichkeiten und den diesen zu Grunde liegenden Systemen weniger gut miteinander harmonisieren).

14 Auch hier benützt Colapietro wieder purpose, vgl. Anm. 8, Anm. der Übersetzerin.

15 Sowohl Peirce wie Colapietro brauchen das ungewöhnliche Wort quietus, das Ruhe, Ende, Todesstoss bedeuten kann. Anm. der Übersetzerin.

16 Peirce spricht in einer undatierten Arbeit, die in den «Collected Papers» unter dem Titel Formen des Bewusstseins publiziert wurde, von den drei Formen des Bewusstseins, die er als primisense, altersense und medisense bezeichnet (vgl. CP 7.551). Dabei legt er sehr viel Wert darauf, dass der primisense, die subjektive Form des Firsts, ein reines Gefühl sei. Auf der Stufe des altersense setzt sich das Bewusstsein dann mit Objekten auseinander, der medisense (Third) verbindet primisense und altersense, Anm. der Übersetzerin.

17 Auch hier benützt Colapietro wieder das Wortpaar cenoscopic/ideoscopic, vgl. Fussnote 12. Anm. der Übersetzerin.

18 Peirce verwendete oft die dem Griechischen nähere Form semeiotic, aber auch, synonym, semiotic. Anm. der Übersetzerin. 
19 Die Übersetzung wird leicht verändert übernommen, das Freud-Zitat durch den ursprünglichen, deutschen Text von Freud ersetzt. Anm. der Übersetzerin.

20 Entgegen der Behauptung von Elisabeth Saporiti, die sie in der Diskussion nach der Präsentation der ersten Version dieses Papiers aufstellte, betrifft dieser Punkt nicht etwas, was nur den frühen Freud angeht und das in späteren Entwicklungen seiner psychoanalytischen Theorie vernachlässigbar wurde (vgl. Wollheim, 1972). Um sicher zu gehen sollte indessen die charakteristische Form der Andersheit zwischen Ich und Über-Ich anhand der Andersheit des Unbewussten selbst analysiert werden.

Zwei Punkte bezüglich der Andersheit des Unbewussten mögen klären helfen, was eine peirceanische Theorie des Unbewussten auszeichnen würde. Erstens sagt Peirce ausdrücklich, dass "wir eine geheime Natur haben, welche wir ebenso wie ihre Inhalte nur aufgrund des Verhaltens, das von ihr bestimmt wird und durch die Phänomene dieses Verhaltens, beurteilen können» (1970, S. 418; CP 5.440). Er fährt fort: «alle werden dem zustimmen (oder alle ausser den extremen Nominalisten), aber anti-synechistische Denker [Peirce ist Anhänger des Gedankens der Kontinuität, er bezeichnet sich selbst als Synechisten. Anm. der Übersetzerin] bringen sich dadurch in künstliche Schwierigkeiten, dass sie die Phänomene falsifizieren, indem sie das Bewusstsein so darstellen, als wäre es - so wie es ist - nur eine Haut, ein losgelöstes Gewebe, das einen unbewussten Bereich der geheimen Natur, des Verstandes, der Seele oder der physiologischen Basis überlagert» (ebd.). Im Gegensatz dazu meidet ein Synechist solche Schwierigkeiten, denn er geht davon aus, dass «dieser Unterschied nur relativ klein ist und man keine scharfe Trennungslinie ziehen kann» (ebd.).

Der zweite Punkt: Peirce' Unterscheidung zwischen zwei Graden der Existenz könnte helfen, einerseits die Andersheit zwischen Unbewusstem und Bewusstsein zu klären, andererseits jene zwischen Ich und Über-Ich. Nach Peirce gibt es zwei Grade der Existenz, nämlich einerseits «eine niedere Art, annähernd ein inneres Sein einfacher Qualität, das indessen existentiell und nicht potentiell ist, [ein Grad der Existenz], der in der Aktivität des Dinges zu sich selbst besteht, eine Art embryohaftes Selbstbewusstsein; und ein höherer Grad [andererseits, V.S.], der im Handeln eines Dinges in Bezug zu allen anderen Dingen desselben Universums besteht, das den Grad seiner Abgeschiedenheit vom Anderen anhand seiner Intensität bemisst» (CP 6.346). Peirce schreibt, dass «ein ganzes Universum solcherWesenheit nur die niederere, oder den internen Grad der Existenz besitzen kann» (ebd.). Während die Andersheit zwischen Ich und Über-Ich am besten in Begriffen des niederen Grades der Existenz gefasst wird, kann diejenige zwischen bewusst Handelndem und seiner wirklichen Welt am besten als höherer Grad der Existenz konstruiert werden. Und zum Schluss: Die Andersheit, zwischen dem Unbewussten und dem Bewusstsein ist eine Andersheit, die jener zwischen Selbst und Welt nahe kommt, obwohl es sich natürlich nicht um eine durchgehende, absolute Andersheit handelt.

21 Ich stimme John J. McDermott (1976) zu, der beteuert, dass die Pragmatisten keine detaillierte Theorie der Verdrängung entwickelt haben, glaube aber, dass es in dieser philosophischen Tradition noch sehr viel mehr Ressourcen dazu gibt, als sogar jene, die mit ihr sympathisieren, bemerkt haben. Bruce Wilshire hat kürzlich bezüglich Dewey eine ähnliche Arbeit in Angriff genommen, wie ich sie mir hier in Bezug auf Peirce vornehme. Damit hat er hat ein Untersuchungsfeld eröffnet, in welchem die Texte der Pragmatisten in einen fruchtbaren Kontakt mit wirkungsvollen Formulierungen aus psychoanalytischer Perspektive gebracht werden. Vgl. sein “Body-Mind and Subconsciousness: Tragedy in Dewey's Life and Work" (1993). 